\title{
Telemonitoring of Blood Glucose
}

\author{
A Prototype Android Application Enhancing the Patient / Health Professional Experience Using Health \\ IT Communication Standards
}

\author{
Matthias Frohner \\ University of Applied Sciences \\ Technikum Wien, Department of \\ Biomedical, Health \& Sports \\ Engineering \\ Wien, Austria \\ frohner@technikum-wien.at \\ Philipp Urbauer \\ University of Applied Sciences \\ Technikum Wien, Department of \\ Information Engineering \& Security \\ Wien, Austria \\ urbauer@technikum-wien.at
}

\author{
Markus Meyer \\ HEALTH - Institute for Biomedicine \\ and Health Sciences, JOANNEUM \\ RESEARCH Forschungsgesellschaft \\ $\mathrm{mbH}$ \\ Graz, Austria \\ markus.meyer@joanneum.at \\ Veronika David \\ University of Applied Sciences \\ Technikum Wien, Department of \\ Biomedical, Health \& Sports \\ Engineering \\ Wien, Austria \\ david@technikum-wien.at
}

\author{
Klaus Donsa \\ HEALTH - Institute for Biomedicine \\ and Health Sciences, JOANNEUM \\ RESEARCH Forschungsgesellschaft \\ $\mathrm{mbH}$ \\ Graz, Austria \\ klaus.donsa@joanneum.at \\ Stefan Sauermann \\ University of Applied Sciences \\ Technikum Wien, Department of \\ Biomedical, Health \& Sports \\ Engineering \\ Wien, Austria \\ sauermann@technikum-wien.at
}

\begin{abstract}
The smartphone, as one of the most personalized digital devices of our time, can play an important role in healthcare related matters since it can route health information gained at the patients' side to the care giving health professionals. One use case is the telemonitoring of blood glucose concentrations from type 2 diabetes patients. Since multiple blood glucose meters for the consumer market exist and health professionals operate different information systems standardized communication interfaces are needed. Specifications for those interfaces are available from the Personal Connected Health Alliance. This work introduces an Android application that is able to communicate with blood glucose monitor devices over Bluetooth Low Energy and forward the received and stored blood glucose readings in form of a HL7 Clinical Document Architecture (CDA) document. The created CDA document conforms to HL7 and IHE specifications and is send encrypted and digitally signed via email according to IHE XDM requirements. It could be shown, that the requirements from different standard defining organizations can be implemented in an Android application using stock Android features.
\end{abstract}

\section{CCS CONCEPTS}

- Applied computing $\rightarrow$ Consumer health; Health care information systems; Health informatics; - Theory of computation $\rightarrow$ Semantics and reasoning;

Permission to make digital or hard copies of part or all of this work for personal or classroom use is granted without fee provided that copies are not made or distributed for profit or commercial advantage and that copies bear this notice and the full citation on the first page. Copyrights for third-party components of this work must be honored.

For all other uses, contact the owner/author(s).

DSAI 2018, June 20-22, 2018, Thessaloniki, Greece

(C) 2018 Copyright held by the owner/author(s).

ACM ISBN 978-1-4503-6467-6/18/06.

https://doi.org/10.1145/3218585.3218678

\section{KEYWORDS}

Mobile application, blood glucose, telemonitoring, communication standards

\section{ACM Reference Format:}

Matthias Frohner, Markus Meyer, Klaus Donsa, Philipp Urbauer, Veronika David, and Stefan Sauermann. 2018. Telemonitoring of Blood Glucose: A Prototype Android Application Enhancing the Patient / Health Professional Experience Using Health IT Communication Standards. In DSAI 2018: 8th International Conference on Software Development and Technologies for Enhancing Accessibility and Fighting Info-exclusion, June 20-22, 2018, Thessaloniki, Greece. ACM, New York, NY, USA, 7 pages. https://doi.org/10.1145/ 3218585.3218678

\section{INTRODUCTION}

Diabetes mellitus (DM) is a growing global disease which highly affects the individual patient and also represents a global health burden with financial impact on national health care systems. In 2013 approximately 382 million people were suffering from diabetes. It is estimated that in 2035 this number will reach 592 million. [1, 12]

It is a chronic illness of the metabolic system leading to high blood glucose (BG) levels. Continuous medical care is required to minimize the risk of acute (e.g. ketoacidosis) and long-term complications (e.g. diabetic foot syndrome, nephropathy, retinopathy, cardiovascular diseases or stroke) [5].

Adhering to therapy in chronic diseases like DM requires active participation and is often very burdensome for patients. In order to document the effect of this treatment the patient is required to take blood glucose readings on a regular basis. Blood glucose meters are available on the consumer market for low prices enabling the enduser to take those readings by them self as basis for therapy. The documentation of blood glucose levels is often done in a pen-paperbased fashion. Since the used devices operate on an electronic level and - most probably - the health professional will operate an electronic record of his/her patients, the electronic break by 
exchanging handwritten documentation is not desirable. Moreover, it represents a safety issue since transcribing read data multiple times (from the display of the glucose meter to a notebook and from the notebook into the electronic documentation system) is a source of failure as stated in [6].

\subsection{Background}

The digital recording and forwarding of data that has been produced at a patient's home by the patient himself/herself is a current topic. These sources of data support decision processes by health professionals and patients. In addition, studies showed that telemonitoring of blood glucose for type 2 diabetes is well accepted and increases the motivation for self-management of the disease [13] and decreases the number of the outpatient visits and hospitalization [4]. Paré et al. [23] published a systematic review based on 24 studies conducted mainly in the United States and Europe, summarizing that telemonitoring of diabetes indicates "a trend towards better glycemic control". Another study conducted by Trief et al. [30] concerning telemonitoring of diabetes, focuses on the perception of elderly patients that got equipped with a "home telemedicine unit" to upload their glucose readings, and enabling the patients to conduct video-conferences with health professionals. Although the patients report that they encountered "computer/equipment" problems, they would recommend such a system to other older adults.

Most devices that are operated at a patient's home are nowadays available with a digital interface for data transfer. However, since those devices are available from a large number of different vendors, a common interface is desirable which would ease the integration process into existing IT-equipment (e.g. patients' smartphones or tablets), i.e. no adaption of the software operated by the patient for each single device is needed. Facing this challenge, the Personal Connected Health Alliance (PCHA) [27] promotes the use of standardized communication protocols over well known and used transport channels (e.g. USB, classic Bluetooth and Bluetooth Low Energy, ZigBee, WiFi) (see Figure 1).

The PCHA specifies how to implement such interfaces between "simple" personal healthcare devices (PHD) with patient bound digital equipment, like the patients' smartphone (Personal Health Gateway), within their Personal Health Device Interface Implementation Guideline [25]. Once the data from the PHD is received on the Personal Health Gateway (PHG) this device can link with other services over wide area networks and forward the data to be consumed by the health professionals' software solutions. Such PCHA conform applications for the interaction between PHD and PHG can be implemented using the available features and software libraries that came with the mobile operating systems [11,24]. The definitions stated by PCHA and the knowledge about the implementation possibilities pave the way for larger scale real world application scenarios. Austria, for example, drafted an architecture to implement telemonitoring based on PCHA specifications [29] (Figure 2).

Since the roll-out of a fully functional infrastructure for telemonitoring and going in operation is not expected to take place in the near future, this paper presents another approach enabling a patient to forward blood glucose readings to supervising health professionals. It must be noted that the approach presented here also aligns with standardization efforts and no proprietor interfaces are implemented (Figure 3).

This prototype implementation is aligned and part of the funded research project INNOVATE [31] that is currently conducted within the research focus Secure Services, eHealth \& Mobility at the University of Applied Sciences Technikum Wien.

\subsection{Bluetooth Low Energy}

Devices supporting Bluetooth Low Energy devices, like Smartphones, Tablets, wearables and PHDs, operate within the ISM radio bands and implement the different Generic Attribute Profiles (GATT) where each profile describes the services, characteristics and attributes a device offers [3]. Standardization is achieved by the Bluetooth Special Interest Group's (SIG) specification of GATT services. One of the GATT services, the Glucose service, is important for the solution described in this work. It defines a set of characteristics that can be queried and contain the blood glucose measurements and relevant context information. Meta-information like information about the blood glucose device can be queried using the device information service.

\subsection{The Clinical Document Architecture}

The Clinical Document Architecture (CDA) is a document standard defined by HL7 [16] and specifies the structure (and partly the content) of medical records and has been approved by ANSI since 2005 . This document standard is based on XML and provides features to include human readable information and machine readable information. The latter is of importance to support semantic interoperability [17] where the receiving computer system is able to further interpret and use the data transfered. This requires structured data and common terminologies for coding the included information. As a medical record, a CDA document contains all information relevant at a certain point in time and provides sufficient information of all the parties involved in the generation of the document, i.e. information about the health professionals and their organizations, patients, policy holder, etc. CDA is a technology that is used by multiple large scale implementations of electronic health record systems [22]. Since CDA documents are XML documents, an enduser will not open the XML directly in order to get information, but the XML file is consumed by an XSLT-transformation based on a stylesheet and then viewed in a web browser. This procedure has the big advantage that by changing the used stylesheet the content can be viewed in different ways and - to a certain extent, depending on the implementation - in different languages. This can be achieved due to the possibility of information being generated out of coded information. For coding, it is desired to use internationally harmonized lists of terminology, like SNOMED-CT, LOINC, or HL7 lists.

\subsection{IHE XDM Profile}

Integrating the Healthcare Enterprise (IHE) is a global, non-profit organization that engages actors in the health system to achieve interoperability of ICT-systems in healthcare. Based on real-world requirements and use cases, IHE defines single profiles where each of those profiles describes how a single use case can be solved 


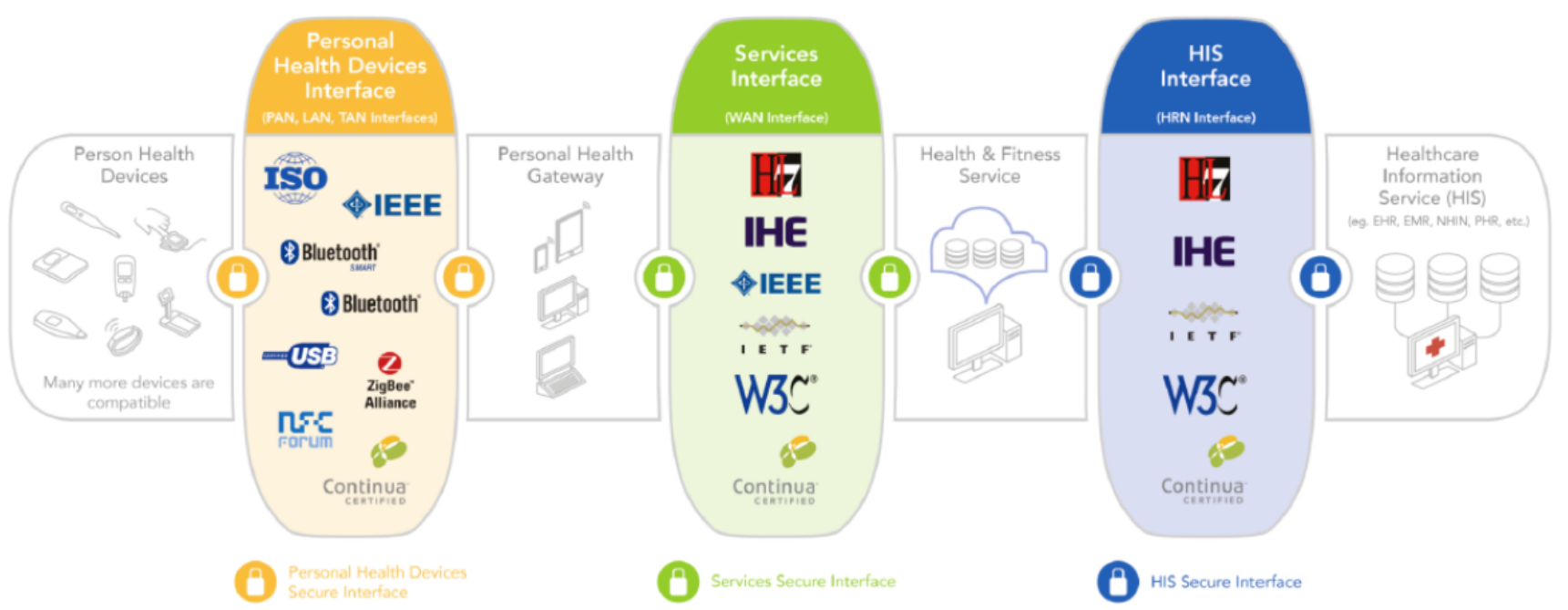

Figure 1: High level architecture as stated by the Personal Connected Health Alliance [26]

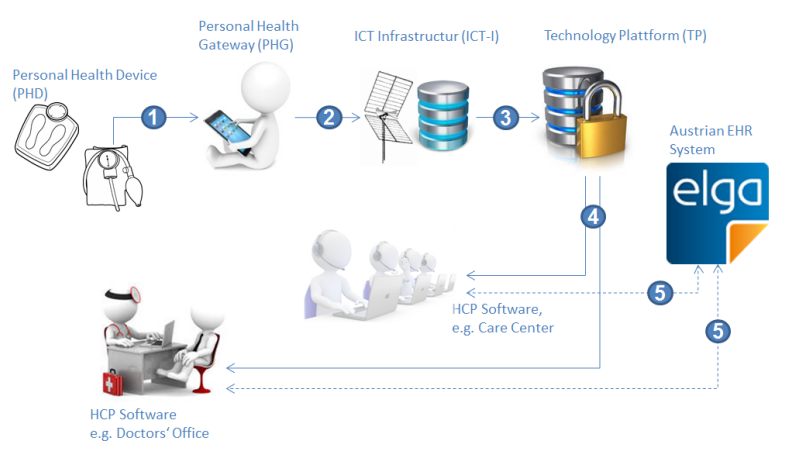

Figure 2: Telemonitoring architecture for Austria [29]. (1) personal/local area network interface between PHD and smartphone/tablet, (2) wide area network interface between smartphone/tablet and back-end service, (3) interface from the back-end service to the technology platform, (4) interface to the health care professionals, (5) interface to the Austria's national health record system named ELGA.

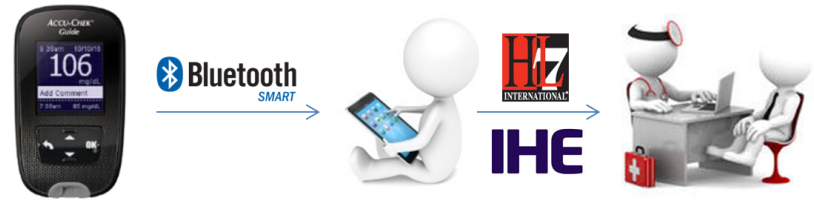

Figure 3: Components and interfaces implemented for the blood glucose telemonitoring use case

technically by applying already existing and used communication standards. In order to achieve interoperability, the identified communication standards get restricted, so that the implementations of the different vendors target the same goal. Specifications of IHE profiles are based on the definition of software modules needed (called actors) and the interaction capabilities with each other (called transactions). One of these defined profiles is the Cross-Enterprise Document Media Interchange (XDM) [18]. This profile states the necessary requirements for sharing information using either USB-memory sticks, CDs or email, where the latter is of importance for this work. According to this profile the shared information can be presented in different ways - one way is the use of HL7's CDA documents. Privacy concerns are addressed by the mandatory use of S/MIME encryption of the email, as well as a digital signature, maintaining confidentiality of the transmitted data and its integrity during the transport.

\section{METHOD}

An Android application capable of communicating with Roche's Accu-Chek ${ }^{\circledR}$ over Bluetooth Low Energy is implemented. Based on the Personal Connected Health Alliance's implementation guideline and specifications of the Bluetooth Special Interest Group, needed services to be implemented for reading blood glucose meter measurements, are identified. The Android application takes advantage of the SQLite database and stores the measurement, meta-information on the measurement (e.g. measurement taken pre-prandial) and the patients' demographic data. The application should enable the user to generate a medical report following HL7's CDA standard specification based on the stored data. This generated report is then sent following IHE's Cross-Enterprise Document Media Interchange [18] specification to the pre-defined health professional via email. Based on this use case, the sequence diagram, shown in Figure 4 was derived. Since the email shall be sent using S/MIME and Androids stock email application does not support sending encrypted and signed mails, the MailDroid [10] email application client is used. This application, together with Crypto Plugin 


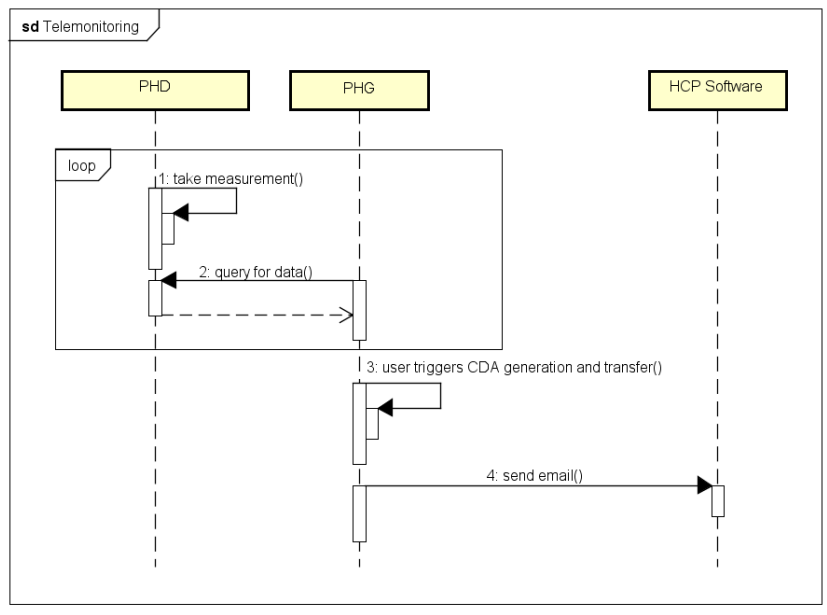

Figure 4: The sequence diagram shows the three actors involved. The blood glucose meter represents the PHD, the smartphone and its application represents the PHG, and the doctors' software represents the Healthcare provider (HCP) application.

[9], enables the S/MIME feature. For testing purposes, the digital certificate for an employee of the University of Applied Sciences Technikum Wien has been installed and configured to be used by MailDroid.

The CDA document is created based on specifications from IHE Patient Care Coordination [19] and HL7 Continuity of Care Documents [14]. Both sources define the structure and the content for coding the patient's vital signs. Using these references, the generated CDA documents follow international harmonized specifications to transport blood glucose information in a human readable and machine readable format. Beside other content that is specified in the before mentioned standards, only the section for coded vital signs are of importance for the implementation described in this work. However, a harmonization with national specification in Austria stated by ELGA's CDA implementation guidelines is desirable. ELGA is already referencing to the vital signs sections of IHE and HL7 in their implementation guidelines for discharge documents $[7,8]$. In order to achieve machine readability, the transported information needs to be assigned to unique codes. In the field of medical IT, code systems like the Systematized Nomenclature of Medicine Clinical Terms (SNOMED CT), Logical Observation Identifiers Names and Codes (LOINC) and HL7 code lists are prominent. For the communication between personal healthcare devices and personal gateway devices ISO/IEEE 11073-10101, the standard for Health informatics - Point-of-care medical device communication Part 10101: Nomenclature, is also relevant. For the ISO/IEEE nomenclature device class, specific extensions are available, like the ISO/IEEE 11073-10417 specifying requirements for communication with blood glucose meters where the specific additional nomenclature is defined. Table 1 gives an overview of relevant codes defined by Bluetooth Low Energy for this work and their mapping to ISO/IEEE and LOINC.

\begin{tabular}{|c|c|c|}
\hline \multicolumn{3}{|c|}{ Concepts used for blood glucose } \\
\hline $\begin{array}{l}\text { BLE Measurement } \\
\text { Context }\end{array}$ & $\begin{array}{l}\text { ISO/IEEE 11073- } \\
10417\end{array}$ & LOINC \\
\hline $\begin{array}{l}\text { [1] Preprandial (be- } \\
\text { fore meal) }\end{array}$ & $\begin{array}{l}\text { [29260] } \\
\text { MDC_CTXT_ } \\
\text { GLU_MEAL_- } \\
\text { PREPRANDIAL }\end{array}$ & $\begin{array}{l}\text { [88365-2] Glucose } \\
\text { [Mass/volume] in } \\
\text { Blood -pre-meal }\end{array}$ \\
\hline $\begin{array}{l}\text { [2] Postprandial (af- } \\
\text { ter meal) }\end{array}$ & $\begin{array}{l}{[29264]} \\
\text { MDC_CTXT_GLU_ } \\
\text { MEAL_ } \\
\text { POSTPRANDIAL }\end{array}$ & $\begin{array}{l}\text { [87422-2] Glucose } \\
\text { [Mass/volume] in } \\
\text { Blood -post meal }\end{array}$ \\
\hline [3] Fasting & $\begin{array}{l}\text { [29268] } \\
\text { MDC_CTXT_GLU_ } \\
\text { MEAL_FASTING }\end{array}$ & $\begin{array}{l}\text { [41604-0] Fast- } \\
\text { ing glucose } \\
\text { [Mass/volume] } \\
\text { in Capillary blood } \\
\text { by Glucometer }\end{array}$ \\
\hline $\begin{array}{l}\text { [4] Casual (snack, } \\
\text { drinks, etc.) }\end{array}$ & $\begin{array}{l}{[29272]} \\
\text { MDC_CTXT_GLU_ } \\
\text { MEAL_CASUAL }\end{array}$ & [?] \\
\hline [5] Bedtime & $\begin{array}{l}{[29300]} \\
\text { MDC_CTXT_GLU_ } \\
\text { MEAL_BEDTIME }\end{array}$ & {$[?]$} \\
\hline
\end{tabular}

Table 1: Blood glucose measurement context. Mapping of coded information from codes used in Bluetooth Low Energy to codes that are available in ISO/IEEE 11073-10417 Health Informatics - Personal health device communication Part 10417: Device specialization - Glucose meter and LOINC. The numbers in squared brackets represent the code, whereas the text following the code represents the clear text representation of the code. For casual and bedtime no corresponding representations have been found in LOINC.

\section{RESULTS}

The developed Android application is capable of receiving blood glucose readings from Roche's Accu-Chek ${ }^{\circledR}$ blood glucose meter. Beside the reading and the physical unit, the timestamp and meta-information (preprandial, postprandial) are transmitted over Bluetooth Low Energy. The received information is stored on the Android device in a SQLite database. When the user decides to send the blood glucose readings to the health professional, values from the database are queried and a transformation process will create the content for the CDA document.

The visualization of the generated CDA document is achieved using a stylesheet, for example the official ELGA stylesheet. Figure 5 shows the table representing the blood glucose readings (this figure shows only a small part of the whole document). This graphic representation is targeted to be read by the patient or the health professional. For machine readability, the same information is coded within corresponding CDA structures. Figure 6 shows the coding of one blood glucose reading in an CDA observation. The single observation contains: 


Vital Signs
Blood Glucose
\begin{tabular}{|llll|}
\hline Value & Meal & Unit & Timestamp \\
120 & postprandial & $\mathrm{mg} / \mathrm{dL}$ & 20.02 .201814 .39 \\
123 & postprandial & $\mathrm{mg} / \mathrm{dL}$ & 20.02 .201818 .17 \\
115 & postprandial & $\mathrm{mg} / \mathrm{dL}$ & $21.02 .201807: 12$ \\
\hline
\end{tabular}

Figure 5: Snipped of the CDA document, visualized using ELGA's official stylesheet, showing the relevant section containing the blood glucose readings. For each reading the physical unit, information concerning the meal and the timestamp is shown in a separate line within the table.

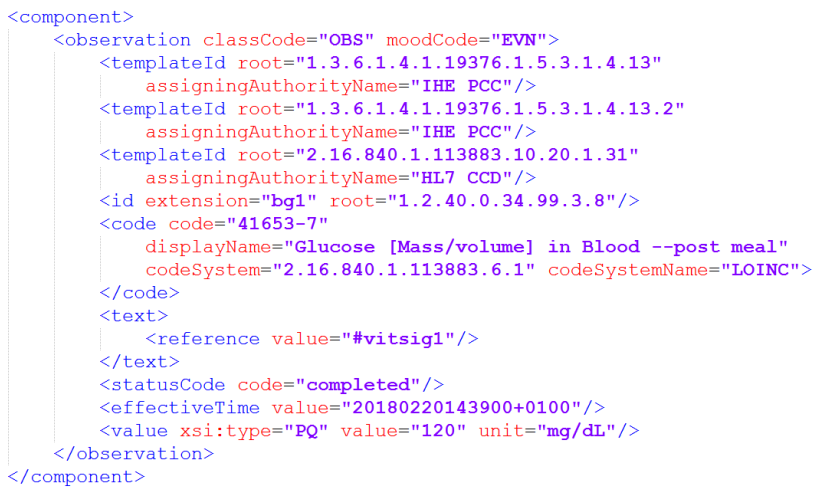

Figure 6: Snipped of the CDA document showing a $C D A$ observation reporting a single blood glucose reading

- a set of templateIds stating conformance to the different observation-templates defined by IHE and HL7.

- The observation/code element represents in a machine readable way the type of the measured parameter, i.e. in the example shown in Figure 6 holds the code for a post-prandial glucose measurement.

- The text element below states a reference to the human readable information. This enables the receiving software system to include the source of the reference (the human readable text) into the database.

- The effectiveTime element contains the date and time information for the blood glucose measurement as received from the blood glucose meter.

- The last element shown in the example is the value element. According to HL7 CDA specification, the data type of this element is from the generic type ANY. Using the xsi:type attribute the flavor of this data type for this observation is expressed. For the blood glucose reading, the data is from type $P Q$ - standing for physical quantity - and the measurement itself and the physical unit are coded in the value and unit element respectively.

Depending on the number of transmitted values, multiple observations are grouped in a CDA organizer.

Based on IHE's specification for cross-enterprise document media interchange, especially the transaction Distribute Document Set on Media [ITI-32] [21] a temporal folder structure is created (see folder structure in Figure 7). This folder structure, including the

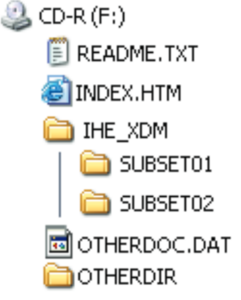

(a)

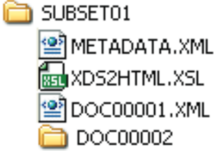

(b)
Figure 7: Folder structure within the ZIP file (based on [21]). (a) General structure of media that shall be transmitted. (b) Structure of the submission set directory, including the CDA document, needed metadata, and the stylesheet to visualize the CDA

generated CDA and the stylesheet, is then compressed in a ZIP file. This ZIP file, together with configured email recipient, email subject and email text, is forwarded to MailDroid via an Android intent. The user can now send the signed and encrypted email.

\section{DISCUSSION}

Currently, the architecture for telemonitoring use cases consisting of a measurement device, a personal health gateway device and the server sided infrastructure is the golden standard. The future might bring the possibility to have personal health devices directly connected to back-end servers due to possible wide area interfaces. In the meantime, devices mapping information from personal/local area networks to wide area networks are needed. One possible infrastructure setup has been shown in this paper. Data is collected and stored locally - at the patients' side - and transmitted as a bulk in form of a medical document. Another approach would be to directly forward received data from the personal health gateway to the health \& fitness services or the healthcare information service. This approach is reflected by the implementation of IHE's Device Enterprise Communication (DEC) profile from the patient care coordination (PCD) technical framework [20]. This profile specifies the transfer of data as either an HL7 message or using HL7 FHIR. Both forms of communication will be triggered by receiving the measurement from the PHD, and most probably not by the user.

The choice for the CDA document structure is based on the ongoing standardization efforts for regional and national healthcare record systems. Such systems require a digital mean to share information across organizational boundaries. The typical approach in harmonizing the shared documents is the definition of implementation guides, specifying how content shall be structured and what terminologies shall be used for coding the content in order to reach machine readability and semantic interoperability. Such standardization efforts consider local requirements, but try to harmonize content on an international level. For this purpose, codes from ISO/IEEE and LOINC have been considered. The decision to code information using the latter is based on the facts that LOINC is very prominent for coding concepts in clinical reports and LOINC codes can be found available for free on their webpage (https://loinc.org). 
CDA provides on the document-level means to code the author of the document. For most use cases the author will be a health professional. Since the content of this document is not created by a person but by the developed Android application, this circumstance is stated within the CDA using the AuthoringDevice class.

The implemented and described application conforms to HL7, PCHA and IHE specification with the single exception that IHE's Audit Trail and Node Authentication actors, as described in [18], are not fully implemented yet. The task of these actors is to log activities during the usage of the system. Hence, it would be required to send a Syslog messages whenever a new email is sent.

The usage of encrypted and signed emails requires digital certificates and the prototype implementation proves that this can be easily achieved. However, the process how patients and doctors get their certificates and how the initial configuration of the email client is done is not solved by this work. This issue might result in usability challenges and will harden the initial installation and set-up procedure with a potential for possible failures. Arnhold et al. [2] present results of an expert-based usability evaluation of diabetes applications available for Android and iOS. These results show, that the "fault tolerance" and efficient management of faults is the most crucial factor for elderly users.

At the time being, the applications facilitates SQLite for persisting measured values gained from the PHDs. Recent work from HL7 resulted in the definition of a functional framework for consumer targeted mobile health application [15], a standard for trail use, that states that personally identifiable information and protected health information shall be stored as encrypted values. Since SQLite is not able to fulfill this conformance criteria at this moment out of the box, other persisting frameworks, like Realm [28], should be considered to be used for future improvements of the application.

Technologies available currently enable the simple telemonitoring solutions and even more complex scenarios. Processes and reimbursement schemes need to be developed and evaluated in order to bring these beneficial and supporting technologies to all stakeholders involved in health care related issues, especially to the patient.

\section{ACKNOWLEDGMENTS}

This project, INNOVATE (project-number 19-06), is funded by the City of Vienna Municipal department 23 Economic Affairs, Labour and Statistics within the program Research at Viennese Universities of Applied Sciences.

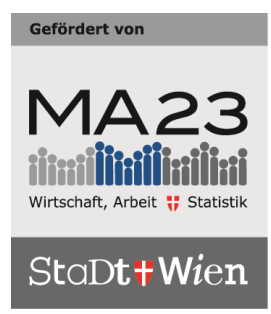

\section{REFERENCES}

[1] American Diabetes Association. 2016. Economic costs of diabetes in the U.S. in 2012. Diabetes Care 39, 7 (2016), 1033-1046. https://doi.org/10.2337/dc12-2625

[2] Madlen Arnhold, Mandy Quade, and Wilhelm Kirch. 2014. Mobile applications for diabetics: a systematic review and expert-based usability evaluation considering the special requirements of diabetes patients age 50 years or older. Fournal of medical Internet research 16, 4 (apr 2014), e104. https://doi.org/10.2196/jmir.2968

[3] Bluetooth SIG. 2014. BLUETOOTH SPECIFICATION Version 4.2 Vol 0: Master Table of Contents \& Compliance Requirements. 0, December (2014), 154.

[4] Claudio Dario, Roberto Toffanin, Francesco Calcaterra, Claudio Saccavini, Panagiotis Stafylas, Silvia Mancin, and Elena Vio. 2017. Telemonitoring of Type
2 Diabetes Mellitus in Italy. Telemedicine and e-Health 23, 2 (2017), 143-152. https://doi.org/10.1089/tmj.2015.0224

[5] Trudi A. Deakin, Catherine E. McShane, Janet E. Cade, and Rhys Williams. 2005. Group based training for self-management strategies in people with type 2 diabetes mellitus. Cochrane Database of Systematic Reviews 2 (2005). https: //doi.org/10.1002/14651858.CD003417.pub2

[6] Klaus Donsa, Peter Beck, Bernhard Höll, Julia K. Mader, Lukas Schaupp, Johannes Plank, Katharina M. Neubauer, Christian Baumgartner, and Thomas R. Pieber. 2016. Impact of errors in paper-based and computerized diabetes management with decision support for hospitalized patients with type 2 diabetes. A post-hoc analysis of a before and after study. International fournal of Medical Informatics 90 (2016), 58-67. https://doi.org/10.1016/j.ijmedinf.2016.03.007

[7] ELGA GmbH. 2017. ELGA CDA Implementierungsleitfäden HL7 Implementation Guide for $C D A \circledast$ R2: Entlassungsbrief (Ärztlich). Technical Report. 1-218 pages.

[8] ELGA GmbH. 2017. ELGA CDA Implementierungsleitfäden HL7 Implementation Guide for $C D A \circledast$ R2: Pflegerischer Entlassungsbrief. Technical Report. 1-94 pages.

[9] LLC Flipdog Solutions. 2018. FlipdogSolution Cryptop Plugin. (2018). http: //flipdogsolutions.com/

[10] LLC Flipdog Solutions. 2018. MailDroid - Email Application. (2018). http: //flipdogsolutions.com/

[11] Matthias Frohner, Philipp Urbauer, Mathias Forjan, Birgit Pohn, Ferenc Gerbovics, Stefan Sauermann, and Alexander Mense. 2012. Development of an android app in compliance with the continua health alliance design guidelines for medical device connectivity in mhealth. Biomedizinische Technik 57, SUPPL. 1 TRACK-N (sep 2012), 997-999.

[12] L. Guariguata, D. R. Whiting, I. Hambleton, J. Beagley, U. Linnenkamp, and J. E. Shaw. 2014. Global estimates of diabetes prevalence for 2013 and projections for 2035. Diabetes Research and Clinical Practice 103, 2 (2014), 137-149. https: //doi.org/10.1016/j.diabres.2013.11.002 arXiv:arXiv:1011.1669v3

[13] Janet Hanley, Peter Fairbrother, Lucy McCloughan, Claudia Pagliari, Mary Paterson, Hilary Pinnock, Aziz Sheikh, Sarah Wild, and Brian McKinstry. 2015. Qualitative study of telemonitoring of blood glucose and blood pressure in type 2 diabetes. BM7 Open 5, 12 (2015), 1-8. https://doi.org/10.1136/bmjopen-2015-008896

[14] Health Level Seven International. 2007. HL7 Implementation Guide : CDA Release 2 - Continuity of Care Document (CCD). Ccd (2007), 111.

[15] Health Level Seven International. 2018. HL7 - Consumer Mobile Health Application Functional Framework, Release 1 - STU Ballot. (2018).

[16] Health Level Seven International. 2018. Structured Documents Group. (2018). http://www.hl7.org/Special/committees/structure/

[17] Healthcare Information and Management Systems Society. 2013. Definition of Interoperability. Himss (2013), 2013. http://www.himss.org/sites/himssorg/files/ FileDownloads/HIMSSInteroperabilityDefinitionFINAL.pdf

[18] IHE International Inc. 2011. IHE IT Infrastructure Technical Framework: Volume 1 (ITI TF-1) - Integration Profiles. Technical Report 8.0. 1-344 pages. http://www.ihe.net/Technical\{_\}Framework/upload/ IHE\{_\}ITI \{_\}TF\{_Rev8-0\{_\}Vol1__\}FT\{_\}2011-08-19.pdf

[19] IHE International Inc. 2011. IHE Patient Care Device (PCD) Technical Framework Volume 2 Transactions. 2 (2011), 1-37.

[20] IHE International Inc. 2012. IHE Patient Care Coordination ( PCC ) Technical Framework Volume 2 Transactions and Content Profiles. 10 (2012).

[21] IHE International Inc. 2014. IHE IT Infrastructure Technical Framework Volume 2b Transactions Part B. 2 (2014).

[22] Millieu Ltd and Time.Lex. 2014. Overview of the national laws on electronic health records in the EU Member States and their interaction with the provision of cross-border eHealth services. March (2014), 34.

[23] Guy Paré, Khalil Moqadem, Gilles Pineau, and Carole St-Hilaire. 2010. Clinical effects of home telemonitoring in the context of diabetes, asthma, heart failure and hypertension: a systematic review. Journal of medical Internet research 12, 2 (jun 2010), e21. https://doi.org/10.2196/jmir.1357

[24] Hyun Sang Park, Hune Cho, and Hwa Sun Kim. 2015. Development of cell phone application for blood glucose self-monitoring based on ISO/IEEE 11073 and HL7 CCD. Healthcare Informatics Research 21, 2 (2015), 83-94. https: //doi.org/10.4258/hir.2015.21.2.83

[25] Personal Connected Health Alliance. 2016. H . 811 Personal Health Devices Interface Design Guidelines, Version 2016 (August 4, 2016). (2016).

[26] Personal Connected Health Alliance. 2017. Fundamentals of Data Exchange Continua. September (2017), 10.

[27] Personal Connected Health Alliance. 2018. Personal Connected Health Alliance. (2018). http://www.pchalliance.org/

[28] Realm. 2018. Realm Database. (2018). https://realm.io/products/realm-database/

[29] Stefan Sauermann and Irina Weik. 2016. eHealth Applications in Austria: Telemonitoring. In 7. Nationaler Fachkongress Telemedizin. Berlin.

[30] Paula M. Trief, Jonathan Sandberg, Roberto Izquierdo, Philip C. Morin, Steven Shea, Rebecca Brittain, Elizabeth Banks Feldhousen, and Ruth S. Weinstock. 2008. Diabetes Management Assisted by Telemedicine: Patient Perspectives. Telemedicine and e-Health 14, 7 (sep 2008), 647-655. https://doi.org/10.1089/tmj. 2007.0107 